\title{
ASSOCIAÇÃO DA FORÇA MUSCULAR RESPIRATÓRIA E O ESTADO NUTRICIONAL DE PACIENTES NEUROLÓGICOS
}

\author{
ASSOCIATION OF RESPIRATORY MUSCLE FORCE AND THE \\ NUTRITIONAL STATUS OF NEUROLOGICAL PATIENTS
}

Liliane Rodrigues da Nóbrega ${ }^{1}$

Ubiraídys de Andrade Isidório ${ }^{2}$

Marta Ligia Vieira Melo ${ }^{3}$

Vitor Engrácia Valenti ${ }^{4}$

Elisangela Vilar de Assis ${ }^{5}$

RESUMO: OBJETIVO: Avaliar a associação entre a força muscular respiratória e o estado nutricional de pacientes neurológicos. MÉTODO: Pesquisa transversal analítica quantitativa. A pesquisa foi realizada na Clínica Escola Integrada da Faculdade Santa Maria- FSM, Cajazeiras, Paraíba, durante o período de abril a maio de 2018. Foram incluídos na pesquisa indivíduos com comprometimento neurofuncional, de ambos os sexos e qualquer idade. Foram excluídos os que possuíam alteração do estado cognitivo e os impossibilitados de realizar a antropometria e a manovacuometria. RESULTADOS: Foram avaliados 12 pacientes de ambos os sexos, onde a maioria se autodeclararam pardas, casadas, com diagnóstico clínico de AVE e sem antecedentes pessoais para doenças cardiovasculares. Observou-se que quando as variáveis da PImáx e PEmáx foram correlacionadas, foi encontrada uma correlação positiva com a circunferência da cintura e a circunferência abdominal no sexo feminino. Enquanto que, essas pressões se correlacionaram negativamente com a prega cutânea subescapular, circunferência abdominal e circunferência da cintura nos homens. Essa relação negativa ainda foi observada com a prega cutânea subescapular, bicipital e a pressão arterial diastólica nas mulheres. CONCLUSÃO: Conclui-se que nos participantes avaliados do sexo feminino, houve uma correlação positiva, entre a

\footnotetext{
${ }^{1}$ Acadêmica do curso de Fisioterapia da Faculdade Santa Maria, Cajazeiras - Paraíba, Brasil. E- mail: lili_rodriguesnobrega@hotmail.com.

2 Mestre em Ciências da Saúde pela Universidade Cruzeiro do Sul - UNICSUL. Docente da Faculdade Santa Maria, Cajazeiras - PB. E-mail: ubiraidys_1@hotmal.com.

${ }^{3}$ Mestre em Saúde Coletiva pela Universidade de Santos - UNISANTOS. Docente da Faculdade Santa Maria, Cajazeiras - PB. E-mail: martaligiafisio@hotmail.com.

${ }^{4}$ Pós-Doutor em Fisiopatologia pela FM/USP. Docente do Departamento de Fonoaudiologia da FFCUNESP/Marília. E-mail: vitor.valenti@gmail.com.

${ }^{5}$ Docente do Curso de Fisioterapia da Faculdade Santa Maria, Cajazeiras - Paraíba, Brasil. E-mail: ely.vilar@hotmail.com.
} 
força muscular respiratória e os parâmetros antropométricos, circunferência da cintura e abdominal.

Palavras chave: Doenças do Sistema Nervoso. Estado Nutricional. Força Muscular Respiratória.

ABSTRACT: OBJECTIVE: To evaluate the association between respiratory muscle strength and the nutritional status of neurological patients. METHOD: Cross-sectional quantitative research. The research was carried out at the Integrated School Clinic of Faculdade Santa Maria - FSM, Cajazeiras, Paraíba, from April to May, 2018. Individuals with neurofunctional impairment of both sexes and any age were included in the study. Those who had altered cognitive status and those who were unable to perform anthropometry and manovacuometry were excluded. RESULTS: Twelve patients of both sexes were evaluated, most of whom declared themselves to be brown, married, with clinical diagnosis of stroke and without a personal history of cardiovascular diseases. It was observed that when the MIP and MEP variables were correlated, a positive correlation was found with waist circumference and waist circumference in females. While, these pressures correlated negatively with the subscapular skinfold, abdominal circumference and waist circumference in men. This negative relation was still observed with subscapular, bicipital skinfold and diastolic blood pressure in women. CONCLUSION: It was concluded that in the female participants, there was a positive correlation between respiratory muscle strength and anthropometric parameters, waist circumference and abdominal circumference, which are related to fat in the abdominal region and, consequently, to the risk for cardiovascular diseases.

Keywords: Nervous System Diseases. Nutritional status. Respiratory Muscular Strength. 


\section{INTRODUÇÃO}

Patologias neurológicas são as doenças do sistema nervoso central e periférico, que incluem desordens do cérebro, da medula espinhal, dos nervos periféricos e da junção neuromuscular. Podendo variar, de anomalias congênitas, acidentes vasculares ou doenças degenerativas, repercutindo nas habilidades físicas e cognitivas, atingindo o estado nutricional, dependendo da localização da lesão (COSTA et al., 2010; SIMONY et al., 2014). Algum transtorno em uma das várias funções do sistema nervoso que são exigidas na alimentação pode afetar a capacidade do indivíduo de cumprir as demandas metabólicas, por resultado da sua incapacidade de manter uma nutrição adequada (LIMA; OLIVEIRA, 2016).

Estudos afirmam que as doenças neurológicas no Brasil e no mundo tem uma grande incidência, afetando um bilhão de pessoas em todo o mundo, segundo a Organização Mundial de Saúde (OMS), essas doenças também incluem, derrames, mal de Parkinson e lesões cerebrais, matando cerca de 6,8 milhões de pessoas por ano, equivalendo a $12 \%$ das mortes globais (GAVIM et al., 2013).

O paciente neurológico tende a apresentar modificações tanto orgânicas como também psíquicas, pelo fato de não aceitar a condição que se encontra. Algumas das mudanças encontradas são alterações sensitivas, na fala, distúrbios cognitivos e visuais, além de déficits na realização de movimentos, que por sua vez, podem restringir o seu desempenho funcional, interferindo no dia-a-dia desses indivíduos (ANDRADE et al., 2010).

Um dos importantes parâmetros observados na prática clínica é a força muscular respiratória, onde os principais responsáveis pelo desempenho da mecânica ventilatória, ou seja, o trabalho respiratório vão ser esses músculos. A utilidade dessa mensuração é para detectar fraqueza da musculatura respiratória e sua gravidade (NASCIMENTO et al., 2012; TAVANA et al., 2015).

Os indivíduos que apresentam alteração de força como consequência da diminuição da massa muscular, podem ter prejuízos na realização das atividades de 
vida diária (AVD's). Porém, a força muscular e habilidades funcionais estão correlacionadas não somente com a massa muscular, como também, ao estado nutricional desse indivíduo (MARTIN; NEBULONI e NAJAS, 2012).

A ausência da capacidade de deambulação e as alterações posturais estão associadas com a redução da força muscular respiratória, pois os músculos tidos como estabilizadores do tronco e cintura pélvica são os acessórios da respiração. Uma alimentação inadequada e má nutrição, relacionada com o quadro clínico da doença, intensifica as complicações respiratórias e motoras (PONTES et al., 2012).

O comprometimento muscular pode ocorrer devido à redução no aporte de substratos energéticos necessários para seu bom funcionamento, como também, por modificações diretas na sua fibra muscular, desde o transporte do impulso neuromuscular até o intercâmbio gasoso, ocasionando redução de resistência e força, como resultado das alterações funcionais e neurofisiológicas. Há uma predisposição de desnutrição protéica calórica em pacientes neurológicos que estão em estado crítico, que aumenta quando não é feita da maneira devida a oferta nutricional (PASSARELLI et al., 2011).

Assim sendo, pesquisas relacionadas ao estado nutricional de pacientes com doenças neurológicas vem crescendo nos últimos anos, pois o estado nutricional apresenta uma importância sobre as respostas fisiológicas do organismo, bem como sobre os danos orgânicos nesse indivíduos. A medição da força dos músculos respiratórios tem uma ampla função, pois permite o diagnóstico de insuficiência respiratória por falência muscular e proporciona o diagnóstico precoce da fraqueza em músculos respiratórios, contribuindo com o profissional da saúde para estabelecer o protocolo de treinamento físico geral e principalmente da musculatura respiratória (SIMONY et al., 2014; HAJJAR, 2007).

Nesse sentido, a presente pesquisa tem por objetivo, fazer uma analise da força muscular respiratória e o estado nutricional de pacientes com alterações neurológicas, tendo em vista que, esse tipo de lesão pode levar a comprometimento físico, como também cognitivo, que pode interferir em uma nutrição adequada, que por sua vez, relacionada com o quadro clínico da doença, poderá potencializar as dificuldades motoras e respiratórias. 


\section{MÉTODO}

Trata se de um estudo do tipo transversal analítico quantitativo.

A coleta foi realizada na Clínica Escola Integrada da Faculdade Santa Maria, localizada na cidade de Cajazeiras, Paraíba, durante o período de abril a maio de 2018, nos turnos matutino e vespertino. Foram avaliados 12 pacientes, atendidos nas disciplinas de Fisioterapia Neurofuncional e Estágio Supervisionado II.

Participaram da pesquisa os pacientes que tinham algum comprometimento neurofuncional, de ambos os sexos, com idade entre 27 e 72 anos, e excluídos os que tinham alteração do estado cognitivo, os impossibilitados de realizar a antropometria e a manovacuometria. Foi utilizado na pesquisa um questionário elaborado pelo próprio pesquisador, constando as seguintes informações: iniciais, idade e sexo, diagnóstico clínico e possíveis doenças/condições associadas a doenças cardiovasculares; verificação dos índices pressóricos (pressão arterial sistólica e diastólica); a avaliação antropométrica, com peso e altura para o cálculo do Índice de Massa Corporal (IMC), circunferência abdominal (CA), circunferência da cintura $(C C)$, circunferência do quadril $(C Q)$, relação cintura/quadril $(R C / Q)$, as pregas cutâneas (bicipital, tricipital e subescapular); e a avaliação da força muscular respiratória.

Foram utilizados estetoscópios e esfigmomanômetros aneroides, para aferição da pressão arterial (PA), sendo realizada uma medida em um dos membros superiores. A classificação adotada foi de acordo com o Seventh Report of the Joint National Committee on Prevention, Detection, Evaluation, and Treatment of High Blood Pressure (JNC 7), em mmHg: normal (sistólica < 120 e diastólica <80); préhipertensão (sistólica entre 120-139 ou diastólica entre 80-89); hipertensão estágio 1 (sistólica entre 140-159 ou diastólica entre 90-99) e hipertensão estágio 2 (sistólica $\geq$ 160 ou diastólica $\geq 100$ )(CONCEIÇÃO et al., 2006).

$\mathrm{Na}$ avaliação antropométrica, foi verificado peso, estando o participante com roupa leves e descalço. Enquanto que, com o estadiômetro foi verificada a altura, na posição ereta. Após a coleta do peso e altura, foi calculado o índice de massa 
corporal $(\mathrm{IMC})$, de acordo com a fórmula IMC = peso $(\mathrm{kg}) /$ altura2 $(\mathrm{cm})$. Para a classificação do IMC foi empregado como ponto de corte, o sugerido pelo World Health Organization (WHO), como: baixo peso (IMC < 18,5); eutrofia (IMC 18,524,99); sobrepeso (IMC 25-29,99) e obesidade (IMC $\geq 30,00$ ) (REZENDE et al., 2006).

$\mathrm{Na}$ CC a fita foi posicionada de forma circundar no ponto médio entre a última costela e a crista ilíaca, na região mais estreia; enquanto que a CA da mesma forma, porém na região da cicatriz umbilical. Para $C Q$ a colocação da fita foi ao redor da região de maior protuberância do quadril. $A R C Q$ foi calculada por meio da divisão da CC pela CQ (MELO et al., 2014; GORZ e TRIBESS, 2009; REZENDE et al., 2006; ACUÑA e CRUZ, 2004).

As pregas cutâneas foram avaliação por meio de um adipômetro, do lado direito do corpo, sendo realizadas três medidas, e sua média o valor adotado. As pregas cutâneas tricipital e bicipital, foram avaliadas na vertical, utilizando como referência para o ponto de medida, o acrômio e o olecrano, na porção posterior e anterior do braço, respectivamente, enquanto que a subescapular, na diagonal, com o ângulo inferior da escápula de referência (TELLES e BARROS, 2003).

Para a avaliação da força muscular respiratória, foi utilizado o manovacuômetro da marca WIKA, com medição de -300 cmH2O a +300 cmH2O. Foi realizada com os indivíduos sentados, utilizando um clipe nasal e sustentando um bocal de maneira firme entre os lábios. Para a mensuração da PImáx, foi solicitada uma expiração pelo bocal, até o volume residual, em seguida, um esforço inspiratório máximo contra a via aérea ocluída. Enquanto que na PEmáx, uma inspiração no bocal até a capacidade pulmonar total, após isso, um esforço máximo expiratório contra a via aérea ocluída.Os participantes realizaram três manobras, com sustentação de pelo menos um segundo, até que foram realizados de maneira adequada três esforços, com intervalos de um minute entre as manobras, e o valor registrado foi o maior (MENEGHETTI et al., 2011).

Para dar início à coleta dos dados o estudo foi submetido inicialmente à análise pelo Comitê de Ética em Pesquisa (CEP) da Faculdade Santa Maria, só após a aprovação, foi dado início de fato a pesquisa, e os participantes foram convidados para participar do estudo, por meio da solicitação da assinatura do 
Termo de Consentimento Livre e Esclarecido (TCLE). Essa pesquisa faz parte da pesquisa intitulada "Associação da força muscular respiratória e o estado nutricional de pacientes neurológicos" que foi aprovada sob o protocolo n 2.516.721(BRASIL, 2012).

Os dados foram analisados no SPSS (versão 25). Além de estatísticas descritivas de frequência relativa e absoluta, e medidas de tendência centra (média e mediana) e de dispersão, foram utilizados teste inferencial de correlação de Spearman (unicaudal). $O$ critério de significância aceito foi de $5 \%$, ou seja, $p \leq 0,05$.

\section{RESULTADOS}

A tabela 1 mostra que houve maioria de pessoas que se autodeclararam pardas, casadas, com diagnóstico clínico de AVE e sem antecedentes pessoais.

Tabela 1. Descrição biodemográfica dos participantes por sexo.

\begin{tabular}{lcccc}
\hline & \multicolumn{3}{c}{ Masculino } & \multicolumn{3}{c}{ Feminino } \\
\cline { 2 - 5 } & \multicolumn{4}{c}{ Frequências } \\
\cline { 2 - 5 } & \multicolumn{1}{c}{$\%$} & $\mathrm{f}$ & $\%$ \\
\hline Etnia & 2 & 28,6 & 2 & 40,0 \\
Branco & 5 & 71,4 & 3 & 60,0 \\
Pardo & & & & \\
Estado civil & 2 & 28,6 & 1 & 20,0 \\
Solteiro & 5 & 71,4 & 3 & 60,0 \\
Casado & 0 & 0,0 & 1 & 20,0 \\
Viúvo & & & & \\
Diagnostico clínico & 4 & 57,1 & 2 & 40,0 \\
AVE & 1 & 14,3 & 0 & 0,0 \\
TCE & 1 & 14,3 & 0 & 0,0 \\
Polineuropatia Periférica & 1 & 14,3 & 0 & 0,0 \\
Esclerose Tuberosa Múltipla & 0 & 0,0 & 1 & 20,0 \\
Siringomielia Cervical & 0 & 0,0 & 1 & 20,0 \\
Distúrbios Vestibulares & 0 & 0,0 & 1 & 20,0 \\
Lesão Crônica do Nervo Ulnar Direito & & & & \\
Antecedentes pessoais & 3 & 42,9 & 4 & 80,0 \\
Sem antecedentes para DCV's & 1 & 14,3 & 1 & 20,0 \\
HAS & 1 & 14,3 & 0 & 0,0 \\
Diabetes & 1 & 14,3 & 0 & 0,0 \\
HAS e diabetes & 1 & 14,3 & 0 & 0,0 \\
HAS, diabetes e doenças renais & & & & \\
\hline
\end{tabular}


Tabela 2. Descrição da antropometria, pressão arterial sistêmica e força muscular respiratória por sexo.

\begin{tabular}{lcccccc}
\hline & \multicolumn{3}{c}{ Sexculino } & \multicolumn{3}{c}{ Feminino } \\
\cline { 2 - 7 } & Média & DP & Mediana & Média & DP & Mediana \\
\cline { 2 - 7 } & 54,28 & 19,68 & 65,00 & 52,20 & 10,94 & 48,00 \\
\hline Idade (anos) & 63,21 & 5,71 & 66,00 & 56,04 & 3,74 & 56,00 \\
Peso (kg) & 1,62 &, 06 & 1,65 & 1,59 &, 06 & 1,600 \\
Altura (cm) & 23,91 & 1,67 & 24,40 & 22,16 & 2,04 & 22,80 \\
IMC (kg/m ${ }^{2}$ ) & 92,00 & 5,80 & 94,00 & 88,20 & 6,05 & 87,00 \\
Circunferência Abdominal (cm) & 88,14 & 6,20 & 89,00 & 80,60 & 4,61 & 81,00 \\
Circunferência da Cintura (cm) & 94,85 & 3,38 & 94,00 & 97,40 & 5,17 & 96,00 \\
Circunferência do Quadril (cm) & 0,92 & 0,05 & 0,93 & 0,82 & 0,07 & 0,84 \\
RCQ (cm) & 7,71 & 2,75 & 7,00 & 11,60 & 1,51 & 11,00 \\
Prega cutânea Bicipital (mm) & 11,42 & 3,35 & 12,00 & 19,80 & 6,72 & 20,00 \\
Prega cutânea Tricipital (mm) & 16,85 & 5,55 & 16,00 & 17,60 & 5,94 & 20,00 \\
Prega cutânea Subescapular (mm) & 128,57 & 16,76 & 120,00 & 114,00 & 5,47 & 110,00 \\
PAS (mmHg) & 84,28 & 9,75 & 80,00 & 78,00 & 14,83 & 80,00 \\
PAD (mmHg) & 61,42 & 32,87 & 60,00 & 56,00 & 26,07 & 60,00 \\
PImáx (cmH2O) & 48,57 & 36,25 & 40,00 & 48,00 & 17,88 & 60,00 \\
PEmáx (cmH2O) & & & & & &
\end{tabular}

A tabela 3 mostra que a PImáx se correlacionou negativamente com a prega cutânea subescapular dos homens, e a prega cutânea bicipital e pressão arterial diastólica nas mulheres, também foi observado que houve correlação positiva apenas no sexo feminino, com a circunferência abdominal e da cintura. Além disso, a PEmáx se correlacionou negativamente com a circunferência abdominal e da cintura masculina, e a prega cutânea subescapular feminina, e ainda positivamente com a circunferência abdominal feminina. Estes resultados foram estatisticamente significativos. 
Tabela 3. Correlação de PImáx e PEmáx entre as variáveis antropométricas e pressão arterial sistêmica divididas pelo sexo.

\begin{tabular}{|c|c|c|c|}
\hline Sexo & Variáveis & PImáx & PEmáx \\
\hline \multirow{13}{*}{ Masculino } & Idade (anos) & $-0,42$ & $-0,21$ \\
\hline & Peso $(\mathrm{kg})$ & 0,06 & 0,30 \\
\hline & Altura $(\mathrm{cm})$ & 0,56 & 0,36 \\
\hline & IMC $\left(\mathrm{kg} / \mathrm{m}^{2}\right)$ & $-0,55$ & $-0,01$ \\
\hline & Circunferência Abdominal (cm) & $-0,38$ & $-0,76^{*}$ \\
\hline & Circunferência da Cintura $(\mathrm{cm})$ & $-0,04$ & $-0,67^{*}$ \\
\hline & Circunferência do Quadril (cm) & 0,15 & $-0,36$ \\
\hline & $\mathrm{RCQ}(\mathrm{cm})$ & $-0,17$ & $-0,60$ \\
\hline & Prega cutânea Bicipital (mm) & $-0,34$ & $-0,37$ \\
\hline & Prega cutânea Tricipital (mm) & $-0,26$ & $-0,47$ \\
\hline & Prega cutânea Subescapular (mm) & $-0,81^{*}$ & $-0,07$ \\
\hline & PAS $(\mathrm{mmHg})$ & $-0,20$ & $-0,19$ \\
\hline & PAD $(\mathrm{mmHg})$ & 0,13 & $-0,21$ \\
\hline \multirow{13}{*}{ Feminino } & Idade (anos) & 0,44 & $-0,16$ \\
\hline & Peso $(\mathrm{kg})$ & $-0,09$ & 0,30 \\
\hline & Altura $(\mathrm{cm})$ & $-0,55$ & 0,06 \\
\hline & IMC $\left(\mathrm{kg} / \mathrm{m}^{2}\right)$ & 0,43 & 0,14 \\
\hline & Circunferência Abdominal $(\mathrm{cm})$ & $0,92^{*}$ & $0,81^{*}$ \\
\hline & Circunferência da Cintura $(\mathrm{cm})$ & $0,81^{*}$ & 0,35 \\
\hline & Circunferência do Quadril (cm) & $-0,28$ & 0,11 \\
\hline & $\mathrm{RCQ}(\mathrm{cm})$ & 0,70 & 0,17 \\
\hline & Prega cutânea Bicipital (mm) & $-0,80^{*}$ & $-0,22$ \\
\hline & Prega cutânea Tricipital (mm) & 0,30 & 0,22 \\
\hline & Prega cutânea Subescapular (mm) & $-0,78$ & $-0,80^{*}$ \\
\hline & PAS $(\mathrm{mmHg})$ & $-0,21$ & 0,10 \\
\hline & PAD $(\mathrm{mmHg})$ & $-0,93^{*}$ & $-0,49$ \\
\hline
\end{tabular}

${ }^{*} p \leq 0,05$ (teste unicaudal); correlação de Spearman.

A tabela 4 mostra que apenas a prega cutânea subescapular se correlacionou com significância estatística e negativa com a PImáx. 
Tabela 4. Correlação de PImáx e PEmáx entre as variáveis antropométricas e pressão arterial sistêmica.

\begin{tabular}{lcc}
\hline \multicolumn{1}{c}{ Variáveis } & PImáx & PEmáx \\
\hline Idade $($ anos $)$ & $-0,21$ & $-0,20$ \\
Peso $(\mathrm{kg})$ & 0,07 & 0,24 \\
Altura $(\mathrm{cm})$ & 0,18 & 0,26 \\
IMC $\left(\mathrm{kg} / \mathrm{m}^{2}\right)$ & $-0,09$ & 0,03 \\
Circunferência Abdominal $(\mathrm{cm})$ & 0,10 & $-0,31$ \\
Circunferência da Cintura $(\mathrm{cm})$ & 0,21 & $-0,37$ \\
Circunferência do Quadril $(\mathrm{cm})$ & $-0,06$ & $-0,17$ \\
RCQ $(\mathrm{cm})$ & 0,20 & $-0,24$ \\
Prega cutânea Bicipital $(\mathrm{mm})$ & $-0,39$ & $-0,26$ \\
Prega cutânea Tricipital $(\mathrm{mm})$ & $-0,04$ & $-0,12$ \\
Prega cutânea Subescapular $(\mathrm{mm})$ & $-\mathbf{0 , 7 9}$ & $-0,25$ \\
PAS (mmHg) & $-0,12$ & $-0,13$ \\
PAD (mmHg) & $-0,28$ & $-0,25$ \\
\hline
\end{tabular}

${ }^{*} p \leq 0,05$ (teste unicaudal); correlação de Spearman.

\section{DISCUSSÃO}

Foram avaliados 12 pacientes de ambos os sexos, onde a maioria se auto declararam pardas, casadas, com diagnóstico clínico de acidente vascular encefálico (AVE) e sem antecedentes pessoais para doenças cardiovasculares. O AVE se destacou como a doença cerebrovascular mais prevalente, apresentando diversas formas, manifestações clínicas e etiologias, além de causar importantes déficits neurológicos, sendo considerado um problema de saúde pública, exigindo enormes demandas em recursos para seu diagnóstico e tratamento (SOUZA et al., 2013; OLIVEIRA et al., 2013).

No presente estudo também foi observado que, quando as variáveis da PImáx e PEmáx foram correlacionadas, foi encontrada uma correlação positiva com a circunferência da cintura $(C C)$ e a circunferência abdominal $(C A)$ no sexo feminino. Enquanto que, essas pressões se correlacionaram negativamente com a prega cutânea subescapular, CC e CA nos homens. Essa relação negativa ainda foi 
observada com a prega cutânea subescapular, bicipital e a pressão arterial diastólica nas mulheres.

Facina; Braga (2013) dizem que o IMC avalia a gordura corporal total, porém de forma indireta, pois não permite a diferenciação do excesso de peso, se ele é oriundo de maior massa de gordura, massa magra, massa óssea ou água corporal, além de que, não permite localizar a adiposidade central. Assim, se faz necessário a associação de outra medida avaliativa para a realização da análise da presença de tecido adiposo. Um importante indicador de gordura abdominal é a circunferência abdominal (CA), sendo relacionada de forma direta com o risco de doenças cardiovasculares. Neste estudo, ambos os sexos obtiveram média de valores dentro da normalidade, se classificando assim, como eutróficos, conforme o sugerido pelo World Health Organization (WHO).

No estudo de Spósit; Santos; Ramires (2002) considera-se que valores de CA superiores a $80 \mathrm{~cm}$ nas mulheres e $94 \mathrm{~cm}$ em homens, associa-se a riscos aumentados para doenças cardiovasculares, como foi observado neste estudo, pois as mulheres obtiveram uma média acima desse valor, enquanto que nos homens, está dentro da normalidade.

Em relação ao peso, nesse estudo as pressões respiratórias máximas não obtiveram correlação com essa variável, porém, segundo o estudo de Costa et al. (2010), pode haver influencia nas pressões, pois o peso está relacionado com à massa muscular, ou seja, alterações no peso podem intervir no desempenho da musculatura respiratória, por poder afetar a massa diafragmática.

A circunferência da cintura (CC) é utilizada como indicador de risco cardiovascular, por ser capaz de revelar acúmulo de gordura intra-abdominal ou visceral, como também, pode ser relacionada com a circunferência do quadril (CQ), estabelecendo a relação cintura/quadril (RCQ), que também é utilizada, como precursor de risco cardiovascular (OLIVEIRA; RODRIGUES, 2016).

Segundo o estudo de Souza et al. (2005) valores superiores a 0,90 em homens, e 0,85 em mulheres de relação cintura/quadril (RCQ), pressupõem risco de desenvolvimento de alterações metabólicas, como observadas também neste estudo, pois os homens obtiveram uma média acima do valor de referência, enquanto que, nas mulheres o valor está dentro do normal. Enquanto que as dobras 
cutâneas ou pregas cutâneas se propõem a avaliar a quantidade de gordura contida no tecido subcutâneo, como também, sua distribuição (TELLES; BARROS, 2003). $\mathrm{Na}$ presente pesquisa as mulheres obtiveram média de valores maiores, quando equiparados aos homens.

Conforme Mendes et al. (2014) a hipertensão arterial sistêmica (HAS) é mais predominante dentro dos fatores para o aparecimento de doenças crônicas nãotransmissíveis, decorrentes do processo de envelhecimento. Se trata da manutenção da pressão arterial em níveis acima de 140 mmHg e 90 mmHg, na sistólica e diastólica, respectivamente. Sendo relacionada com fatores como sexo, idade e raça, hereditariedade, além do tabagismo, sedentarismo, obesidade, estresse, dislipidemia e uma dieta inadequada. Além de aumentar o risco para doenças como AVE. Analisando os sexos, e levando em consideração a classificação adotada de acordo com o Seventh Report of the Joint National Committee on Prevention, Detection, Evaluation, and Treatment of High Blood Pressure (JNC 7), pela média de valores, as mulheres se classificaram como normal, enquanto os homens, como pré- hipertensão.

No estudo de Souza et al. (2013) o prognóstico de pacientes com AVE pode ser afetado pelo estado nutricional, tendo em vista que a desnutrição afeta de $8 \%$ a $16,3 \%$ dos pacientes na admissão hospitalar e aumenta com o tempo de hospitalização. Além de estar associada a complicações como infecção, sepse, úlcera de pressão, tromboembolismo pulmonar, aspiração, pneumonia, hipoalbuminemia grave, hemorragia gastrintestinal e disfagia, maior uso da nutrição enteral e tempo de hospitalização.

De acordo com Caruso et al. (2015) os valores de referência estabelecidos para PImáx em diferentes populações, tem como limite inferior da normalidade de 60 $\mathrm{cmH} 2 \mathrm{O}$ para mulheres e de $80 \mathrm{cmH} 2 \mathrm{O}$ para homens, e para PEmáx, $120 \mathrm{cmH} 2 \mathrm{O}$ para mulheres e de $150 \mathrm{cmH} 2 \mathrm{O}$ para homens. No presente estudo, em ambos os sexos, foi observado valores de PImáx e PEmáx abaixo do limite de normalidade.

Mafalda et al. (2014), em seu estudo, observaram que pacientes com AVE apresentam alteração da força muscular respiratória (FMR), sendo verificado em hemiparéticos, uma redução de $28 \%$ da PImáx e $20 \%$ da PEmáx, após a avaliação dos músculos respiratórios. Ainda foi visto que, a baixa da PImáx nos indivíduos 
avaliados, pode estar associada à fraqueza da musculatura abdominal e torácica, em ambos os sexos, com médias de PImáx abaixo do normal, de 13 $\pm 10,51 \mathrm{cmH} 20$ para o sexo feminino e de $7,43 \pm 2,76 \mathrm{cmH} 2 \mathrm{O}$ para o masculino.

Simões et al. (2007) ainda destacam que, a idade influencia de maneira significativa e de forma progressiva nos valores da PImáx e PEmáx, em ambos os sexos. E quando os valores são comparados com homens e mulheres da mesma faixa etária, entre 40 a 89 anos, foram observados valores significativamente menores nas mulheres. Sua redução é possivelmente considerável na situação clínica dos pacientes idosos, pois as alterações estruturais que ocorrem durante esse processo, podem provocar danos no músculo diafragma, na sua curvatura, onde sua capacidade de gerar força poderá ser afetada, permitindo assim concluir, que a idade e o sexo podem influenciar diretamente na FMR.

Da mesma forma, Almeida; Bertucci; Lima (2008) observaram em seu estudo, que as pressões respiratórias máximas atingem valores máximos, quando adulto jovem e vão reduzindo, de maneira progressiva com o avançar da idade, além disso, observaram valores de PImáx e PEmáx maiores no sexo masculino, quando equiparado ao sexo feminino. Concluindo que, existem fatores que influenciam na inconstância desses valores, de acordo com o sexo, pelas diferencias corporais, com a idade, por existir mudanças na elasticidade pulmonar, caixa torácica e redução de massa e força muscular, além do peso e altura de cada indivíduo.

Taveira; Teixeira; Domingues (2013) apontam que uma avaliação sistemática e simples dos parâmetros respiratórios pode ser útil na detecção de forma precoce da redução da FMR, destacando sua importância como um processo preventivo, tendo em vista que, em seu estudo, foi observada uma redução desses parâmetros em pacientes com Esclerose Múltipla, quando comparado com indivíduos saudáveis, com diminuição das pressões respiratórias máximas.

Como limitações desse estudo pode-se destacar a dificuldade em convidar os pacientes para participarem da pesquisa, pelas intercorrências como, conflito nos horários dos seus atendimentos e os da realização da pesquisa, além de suas faltas, reduzindo a frequência ao atendimento, o que reduziu o número de participantes da pesquisa. E desta forma, pode ter contribuído para a não observação de dados 
claros e pertinentes a respeito de uma possível associação entre a força muscular respiratória e o estado nutricional em pacientes neurológicos.

\section{CONCLUSÃO}

Após a análise, verificou-se que houve correlações significativas entre a FMR e alguns parâmetros antropométricos, como circunferência da cintura e circunferência abdominal no sexo feminino, que estão relacionadas com gordura na região abdominal, e consequentemente, com o risco para doenças cardiovasculares. Desta forma, pôde ser visto que pacientes neurológico são vulneráveis a apresentar alteração do seu estado nutricional, seja por qualquer alteração física ou cognitiva, levando em consideração a localização e o que a lesão irá afetar, e quando esses fatores associados ao processo natural do envelhecimento poderão agravar o seu estado e potencializar as dificuldades motoras e respiratórias, incluindo a força muscular respiratória.

Portanto, torna-se necessário a realização de novos estudos, ampliando o tamanho da amostra, para que possam identificar e esclarecer a associação entre a força muscular respiratória e o estado nutricional de pacientes neurológicos.

\section{REFERÊNCIAS BIBLIOGRÁFICAS}

ACUÑA, K; CRUZ, T. Avaliação do estado nutricional de Adultos e Idosos e situação nutricional da população Brasileira, Arquivos Brasileiros de Endocrinologia \& Metabologia, v. 48, n. 3 , p. $345-361,2004$.

ALMEIDA, I. P.; BERTUCCI, N. R.; LIMA, V. P. Variações da pressão inspiratória máxima e pressão expiratória máxima a partir da capacidade residual funcional ou da capacidade pulmonar total e volume residual em indivíduos normais. Revista O Mundo da Saúde, v. 32, n. 2, p. 176-182, 2008.

ANDRADE, S. M. et al. Independência funcional e qualidade de vida em pacientes com sequelas neurológicas: a contribuição de um grupo terapêutico interdisciplinar. Revista Interdisciplinar de Estudos da Cognição, v. 15, n. 2, p. 155-164, 2010.

BRASIL. Ministério da Saúde. Conselho Nacional de Saúde. Comissão Nacional de Ética em Pesquisa Resolução n. 466/12, de 12 de dezembro de 2012. Brasília: Ministério da Saúde, 
2012.

CARUSO, P. et al. Métodos diagnósticos para avaliação da força muscular inspiratória e expiratória*. Jornal Brasileiro de Pneumologia, v. 41, n. 2, p. 110-123, 2015.

CONCEIÇÃO, T. V. et al. Valores de Pressão Arterial e suas Associações com Fatores de Risco Cardiovasculares em Servidores da Universidade de Brasília. Arquivos Brasileiros de Cardiologia, v. 86, n.1, p. 26-31, 2006.

COSTA, I. et al. Terapia nutricional em doenças neurológicas - Revisão de literatura. Revista neurociências, v. 18, n. 4, p. 555-560, 2010.

COSTA, D. et al. Novos valores de referência para pressões respiratórias máximas na população brasileira*. Jornal Brasileiro de Pneumologia, v. 36, n. 3, pp. 306-312, 2010.

FACINA, V. B.; BRAGA, J. G. L. Perfil antropométrico de adolescentes do Recôncavo da Bahia. Revista Ciência em Extensão, v.9, n.3, p.21-33, 2013.

GAVIM, A. E. O. et al. A influência da avaliação fisioterapêutica na reabilitação neurológica. Revista Saúde em Foco, n. 6, p. 71-77, 2013.

GORZ, F. B.; TRIBESS, S. Circunferência da cintura ou do abdome: qual utilizar para mensurara gordura visceral? Revista Brasileira de Nutrição Clínica, v. 25, n. 3, p. 233-237, 2009.

HAJJAR, N. E. Avaliação da força muscular respiratória em idosos. Revista Pleiade, v. 1, n. 1, p. 95-112, 2007.

LIMA, A. C. G.; OLIVEIRA, M. C. Avaliação nutricional de pacientes neurológicos submetidos à terapia nutricional enteral em uma unidade de terapia intensiva. Revista Brasileira de Nutrição Clínica, v. 31, n. 2, p. 112-117, 2016.

MAFALDA, L. et al. Perfil respiratório de pacientes acometidos por acidente vascular encefálico. Revista Saúde Integrada, v.7, n. 13-14, p. 153-172, 2014.

MARTIN, F. G.; NEBULONI, C. C.; NAJAS, M. S. Correlação entre o estado nutricional e força de preensão palmar em idosos. Revista Brasileira de Geriatria e Gerontologia, v. 15, n. 3, p. 493-504, 2012.

MENDES, G. S. et al. Prevalência de hipertensão arterial sistêmica em idosos no Brasil entre 2006 e 2010. Revista Brasileira de Medicina de Família e Comunidade, v. 9, n. 32, p. $273-$ 278, 2014.

MELO, A. P. F. et al. Métodos de estimativa de peso corporal e altura em adultos hospitalizados: uma análise comparativa Revista Brasileira de Cineantropometria e Desempenho Humano, v. 16, n. 4, p. 475-484, 2014.

MENEGHETTI, C. H. Z. et al. Avaliação da força muscular respiratória em indivíduos acometidos por Acidente Vascular Cerebral. Revista Neurociências, v. 19, n. 1, p. 56-60, 2011.

NASCIMENTO, R. A. et al. Valores encontrados e preditos para as pressões respiratórias máximas de crianças brasileiras. Revista Brasileira de Crescimento e Desenvolvimento Humano, v. 22, n. 2, p. 166-172, 2012.

OLIVEIRA, A. R. S. et al. Avaliação de pacientes com acidente vascular cerebral acompanhados por programas de assistência domiciliária. Revista da Escola de Enfermagem da USP, v. 47, n. 5, p. 1147-53, 2013. 
OLIVEIRA, L.F.; RODRIGUES, P. A. S. Circunferência de cintura: protocolos de mensuração e sua aplicabilidade prática. Revista de Nutrição e Vigilância em Saúde, v. 3, n. 2, p. 90-95, 2016.

PASSARELLI, R. C. V. et al. Avaliação da força muscular inspiratória (PImáx) durante o desmame da ventilação mecânica em pacientes neurológicos internados na unidade de terapia intensiva. Revista Fisioterapia e Pesquisa, vol.18, n.1, p.48-53, 2011.

PONTES, J. F. et al. Força muscular respiratória e perfil postural e nutricional em crianças com doenças neuromusculares. Revista Fisioterapia em movimento, v.25, n.2, p.253-261, 2012.

REZENDE, F.A. C. et al. Índice de Massa Corporal e Circunferência Abdominal: Associação com Fatores de risco Cardiovascular. Arquivos Brasileiro de Cardiologia, v.87, n.6, p.728734, 2006.

SIMÕES, R. P. et al. Influência da idade e do sexo na força muscular respiratória. Fisioterapia e Pesquisa, v.14, n.1, p. 36-41, 2007.

SIMONY, R. F. et al. Caracterização do estado nutricional dos pacientes neurológicos com mobilidade reduzida. Revista Brasileira de Crescimento e Desenvolvimento Humano, v. 24, n. 1, p. 42-48, 2014.

SOUZA, A. F. et al. Análise do Índice de Massa Corporal e Relação Cintura/Quadril dos Participantes do Projeto Academia Escola UCDB. Revista Multitemas, n. 32, p. 23-31, 2005.

SOUZA, A. L. et al. Unidade de cuidado integral ao acidente vascular cerebral agudo e evolução nutricional dos pacientes. Revista Brasileira de Nutrição Clínica, v. 28, n. 2, pp. 98102, 2013.

SPÓSITO, A.C.; SANTOS, R. D.; RAMIRES, J. A. F. Avaliação do Risco Cardiovascular no Excesso de Peso e Obesidade. In: SANTOS, R.cD.; TIMERMAN, S.; SPÓSITO, A.C. Diretrizes Para Cardiologistas Sobre Excesso de Peso e Doença Cardiovascular dos Departamentos de Aterosclerose, Cardiologia Clínica e FUNCOR da Sociedade Brasileira de Cardiologia. Arquivos Brasileiro de Cardiologia, v.78, p. 6-7, 2002.

TAVANA, S. et al. Effect of Dialysis on Maximum Inspiratory and Expiratory Pressures in End Stage Renal Disease Patients. Journal Tanaffos, v. 14, n. 2, p. 128-133, 2015.

TAVEIRA, F. M.; TEIXEIRA, A. L; DOMINGUES, R. B. Early respiratory evaluation should be carried out systematically in patients with multiple sclerosis. Arquivo de Neuropsiquiatria, v.71, n.3, p.142-145, 2013.

TELLES, R. K.; BARROS, A. A. F. O uso da antropometria como método de avaliação da composição corporal em pediatria. Revista de Ciências Médicas, v. 12, n. 4, p. 351-363, 2003. 